\title{
Tyrosine kinase inhibitors significantly improved survival outcomes in patients with metastatic gastrointestinal stromal tumour: a multi-institutional cohort study
}

\author{
A. Deruchie Tan BSc, ${ }^{*}$ K. Willemsma BSc, ${ }^{*}$ A. MacNeill MD, ${ }_{1}^{+}$K. DeVries MSc, ${ }^{\neq}$A. Srikanthan MD MHSc, ${ }^{*}$ \\ C. McGahan $\mathrm{MSc}_{1}^{\neq}$T. Hamilton $\mathrm{MD}_{1}^{\dagger}$ H. Li BSc, ${ }^{*}$ C.D. Blanke MD, ${ }^{\S}$ and C.E. Simmons MD MSc ${ }^{*}$
}

\begin{abstract}
Background The real-world impact of tyrosine kinase inhibitors (TKIs) in clinical practice for gastrointestinal stromal tumour (GIST) has not been extensively reported. We sought to assess how outcomes have changed over the eras and to evaluate the effect of access to imatinib and sunitinib on survival in patients with unresectable or metastatic GIST in British Columbia.
\end{abstract}

Methods Patients with metastatic or unresectable GIST were allocated to one of three eras: pre-2002, 2002-2007, and post-2007 based on treatment availability (pre-imatinib, post-imatinib, and post-sunitinib). Overall survival (OS) and progression-free survival (PFS) were compared between eras. Univariate and multivariate analyses were performed to determine the effects of tumour, patient, and treatment characteristics on survival outcomes.

Results Of 657 patients diagnosed with GIST throughout British Columbia during 1996-2016, 196 had metastatic disease: 23 in the pre-imatinib era, 67 in the post-imatinib era, and 106 in the post-sunitinib era. A significant increase in os, by 53.6 months ( $p=0.0007)$, and PFs, by 29.1 months ( $p=0.044)$, was observed after the introduction of imatinib. The introduction of sunitinib did not significantly affect os or PFS.

Conclusions Implementation of TKIs has drastically improved survival outcomes for patients with metastatic GIST by up to 4.55 years in the real-world setting. Our study demonstrates that implementation of TKIs in clinical practice has outperformed their benefit predicted in clinical trials.

Key Words GIST, gastrointestinal stromal tumour, tyrosine kinase inhibitors, imatinib, sunitinib, sarcoma, realworld data

\section{INTRODUCTION}

Gastrointestinal stromal tumour (GIST) is the most common type of mesenchymal neoplasm of the digestive tract, but accounts for less than $1 \%$ of gastrointestinal tumours and $5 \%$ of all sarcomas ${ }^{1,2}$. Over time, the understanding of this malignancy has evolved significantly. Before 2000, GISTs were not recognized as a separate clinicopathologic entity; they were initially undifferentiated from leiomyomas, leiomyoblastomas, and other gastrointestinal sarcomas ${ }^{1}$. In general, GISTs respond poorly to conventional chemotherapeutic agents, with a response rate of less than $10 \%^{3}$.
The role of radiotherapy is limited, and radiotherapy has historically produced little response ${ }^{4,5}$. Surgical resection remains the standard of care for localized GIST, and it is the only available curative treatment ${ }^{3}$. Historically, if the tumour was deemed technically unresectable, or if patient factors prohibited resection, alternative treatment modalities were predominantly supportive in nature. That approach has, however, been revolutionized with the advent of targeted therapy.

In 1998, work by Hirota etal. ${ }^{6}$ led to the ground-breaking discovery that upward of $95 \%$ of GISTs express KIT (CD117) by immunohistochemistry, allowing for more accurate 
diagnoses $^{7}$. They also discovered that a gain-of-function mutation in the intracellular domain of KIT would lead to constitutive activation of its tyrosine kinase activity without binding of the appropriate ligand, stem-cell factor. That finding led to the eventual understanding of the driving role of KIT in the development and proliferation of GISTs, allowing for the use of targeted therapies. Activation of KIT leads to phosphorylation of secondary signalling molecules (MAPK, STAT, PIP3/AKT), potentially altering gene transcription, cell cycle regulation, and apoptotic mechanisms? ${ }^{7}$. In 2003, researchers discovered that GISTs lacking a mutation in KIT might be driven by a mutation in platelet-derived growth factor $\alpha$ (PDGFRA $)^{1,3,8}$. Approximately $85 \%$ of GISTs contain a mutation in KIT or PDGFRA, with the remaining $15 \%$ being deemed wild-type ${ }^{1}$.

On 1 February 2001, imatinib mesylate (Gleevec: Novartis, Basel, Switzerland) received accelerated approval by the U.S. Food and Drug Administration for the treatment of advanced or metastatic $\mathrm{GIST}^{9}$. Imatinib exerts its antineoplastic effects by selectively occupying the ATP binding pocket of KIT and PDGFRA, thus preventing downstream signalling and ultimately inhibiting tumour cell survival and proliferation ${ }^{1,3}$. In 2008, imatinib received full approval for the treatment of KIT-positive unresectable or metastatic GIST. That approval was based on the marked improvement in survival outcomes observed in a combined analysis of 1640 patients from two randomized phase III trials performed by the European Organisation for Research and Treatment of Cancer and swOG, with os improving to nearly 50 months from 18 months $^{9-12}$. Imatinib was first made available for use at BC Cancer on 1 July $2002^{13}$.

It is recognized that up to $80 \%$ of GISTs will eventually develop resistance to imatinib through the acquisition of secondary mutations in KIT, warranting adoption of nextline therapy ${ }^{14,15}$. On 26 January 2006, sunitinib maleate (Sutent: Pfizer, New York, NY, U.S.A.) received approval from the U.S. Food and Drug Administration as a treatment for patients with GIST after disease progression on or intolerance to imatinib ${ }^{5}$. Sunitinib works through molecular interactions similar to those for imatinib to inhibit multiple receptor tyrosine kinases, including KIT, PDGFRA, and vascular endothelial growth factor receptor ${ }^{5,14}$. Approval of sunitinib was based on an international multicentre randomized double-blind placebo-controlled phase III clinical trial that demonstrated an improvement by a factor of 4 in time to progression (27.3 weeks vs. 6.4 weeks $)^{16}$. Sunitinib was approved for use in Canada in May 2006 and was funded for use at BC Cancer on 1 August $2007^{17}$. In 2013, upon the presentation of phase III evidence of improved PFS (4.8 months vs. 0.9 months) ${ }^{18}$, the U.S. Food and Drug Administration approved regorafenib as a third-line TKI after the failure of imatinib and sunitinib for patients with unresectable GIST. In addition, since 2015, a flurry of studies have assessed the efficacy of newer TKIs in the treatment of metastatic GIST beyond the second line.

The effect of multiple TKIs on real-world outcomes for patients with metastatic or unresectable GIST has not been extensively reported. The largest registry study to date included patients diagnosed with GIST and treated between 2007 and 2011, and did not significantly address the effect of using second- or subsequent-line TKIs such as sunitinib ${ }^{19}$.
Because clinical trials often have strict inclusion criteria, the extrapolation of outcomes to clinical practice can result in variability of efficacy appreciated in an unselected non-trial population. In other disease sites, clinical trial results have been shown to potentially overestimate expected outcomes $^{20-22}$. We set out to assess the effect on survival outcomes of the availability of multiple TKIs for non-trial patients with metastatic or unresectable GIST attending multiple centres in the province of British Columbia.

\section{METHODS}

\section{Study Design}

In a retrospective cohort study, we assessed the effect of TKI availability on outcomes in patients diagnosed with metastatic GIST in British Columbia between 1996 and 2016. Patients with a histologically confirmed diagnosis of GIST between the years 1996 and 2016 were identified through the BC Cancer Registry and BC Cancer's Sarcoma Outcomes Unit, which collects data from all 5 BC Cancer institutions in the province (Vancouver, Victoria, Fraser Valley, Kelowna, and Prince George). Individual chart review was conducted to extract data. Patients with unresectable or metastatic disease were included in the study. Patients were excluded if they had participated in a clinical trial for treating their GIST during the study period or if data were insufficient to determine treatments received or stage of disease (Figure 1).

Tumour characteristics were recorded, including date of initial diagnosis, date of diagnosis of metastatic disease, tumour size at initial diagnosis, and location of the primary tumour. Primary sites were categorized into three groups: gastric, small intestine, or other.

\section{Treatment}

Dates of initiation and discontinuation of systemic therapy, type of systemic therapy, dose received, and timelines of administered systemic therapies were collected and recorded. Dates of progression were recorded as the day on which the treating oncologist first mentioned definitive progression warranting adoption of next-line systemic therapy on the patient's chart. Information about the reason for discontinuation (or non-initiation) of antineoplastic medication was recorded. Cause of death was extracted from the registry data and cross-referenced with a full chart review. For all patients still living at the time of data analysis, date of last contact was recorded to ensure adequate follow-up duration.

Patients were allocated to one of three treatment eras based on their date of diagnosis for metastatic disease: pre-imatinib (pre-2002), post-imatinib (2002-2007), and post-sunitinib (post-2007). The dates on which imatinib (1 July 2002) and sunitinib (1 August 2007) were made available in British Columbia were used as the start dates for the respective era cohorts.

\section{Mitotic Activity}

Pathology reporting of mitotic activity has varied greatly through the years, because no standardized method of reporting had been adopted until recently. To standardize the 
reported values for the present study, the values collected for mitotic activity were converted to mitotic figures per $5 \mathrm{~mm}^{2}$. The conversion factor used was 10 high-power fields (HPFs) per $1 \mathrm{~mm}^{2}$. Mitotic activity reported in mitoses per $n$ HPFs was first converted by dividing $n$ by 10 to determine the number of square millimeters in the reported field of view. That new value was then multiplied by $5 \mathrm{~mm}^{2}$, and the resulting fraction was evaluated to determine the number of mitotic figures per $5 \mathrm{~mm}^{2}$.

\section{Outcomes Analysis}

The primary outcome of interest was os, which was calculated from the time of initial metastatic diagnosis to the date of death or most recent follow-up. Progression-free survival was calculated from the time of initial metastatic diagnosis to the first documented occurrence of progression (defined as a worsening of disease warranting adoption of next-line therapy).

Using the Kaplan-Meier method, survival curves were generated from the collected data. The effects of tumour, patient, and treatment characteristics were tested through univariate analysis using the log-rank test. A $p$ value less than 0.05 was considered to be significant. Multivariate analysis was carried out using Cox regression analysis in the IBM SPSS Statistics software application (version 23: IBM, Armonk, NY, U.S.A.).

\section{RESULTS}

\section{Demographics}

Between 1996 and 2016, 697 patients with a histologically confirmed diagnosis of GIST were identified from 5 different institutions across British Columbia. Of those 697 patients, 196 (28\%) either presented with, or developed, unresectable or metastatic disease during the study period

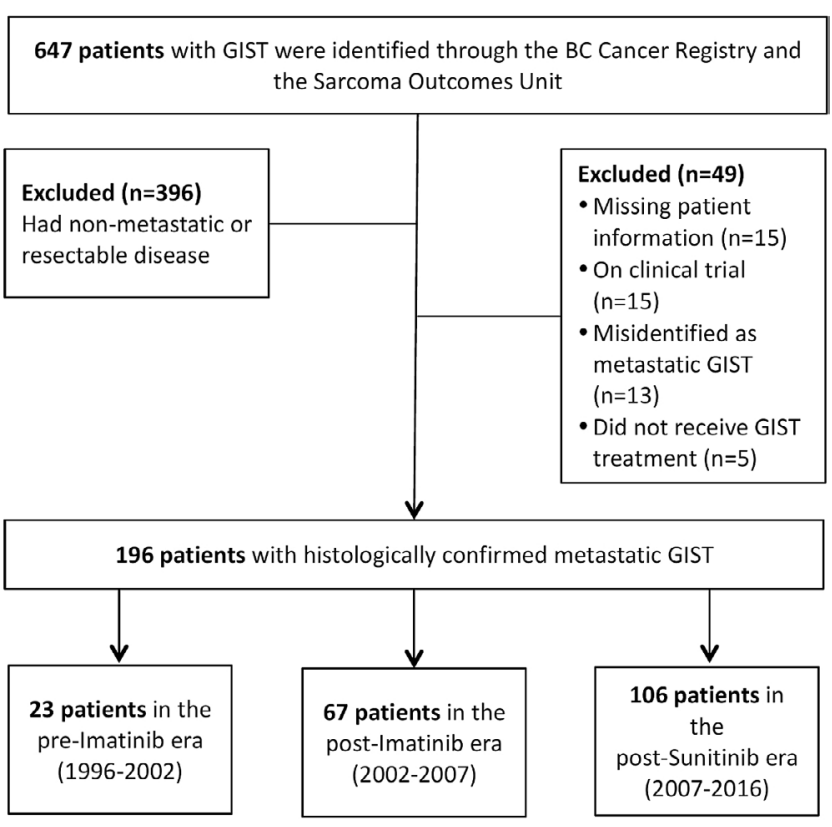

FIGURE 1 CONSORT diagram showing the process of patient selection for the study. GIST = gastrointestinal stromal tumour. and were included in the study population. Based on the time of diagnosis of metastatic disease, 23 patients (12\%) were diagnosed in the pre-imatinib era; 67 (34\%), in the post-imatinib, pre-sunitinib era; and 106 (54\%), in the post-sunitinib era. Table I summarizes the demographics for each era's cohort. Median age at metastatic diagnosis was 64.2 years for the overall group, and the median age was consistent across the eras. The study population consisted of 122 men (62\%) and 74 women (38\%). The most common primary sites were the stomach (75 patients, 38\%) and small intestine (70 patients, $36 \%$ ). The remaining 51 patients $(26 \%)$ had another primary gastrointestinal site. Tumour size at initial diagnosis was reported for 190 patients $(96.9 \%)$, among whom, $162(85.3 \%)$ had a tumour larger than $5 \mathrm{~cm}$ in the greatest dimension. Overall, 93 patients (47\%) presented with metastatic or unresectable disease at the time of initial diagnosis; the remaining 103 patients developed metastatic disease. Expression of KIT protein was tested and reported for 179 patients (91\%), with 173 of those samples (97\%) staining positive for the CD117 antibody. Of the 17 patients with unknown KIT expression, 14 (82\%) belonged to the pre-imatinib era, when CD117 immunostaining was not a standard diagnostic method for GISTs. Immunoreactivity for PDGFRA was not routinely tested in this population.

\section{Outcomes}

Median os improved from 7.8 months [95\% confidence interval (CI): 5.6 months to 14.0 months] in the pre-imatinib era to 61.4 months (95\% CI: 45.0 months to 73.3 months; $p=$ 0.0007 ) in the post-imatinib era, representing a 53.6-month improvement. It increased slightly further from 61.4 months in the post-sunitinib era to 62.2 months (95\% CI: 50.9 months to 86.3 months; $p=$ nonsignificant; Figure 2).

For the patients overall, median PFs increased from 4.8 months (95\% CI: 3.9 months to 14.4 months) in the

TABLE I Patient demographics and tumour characteristics

\begin{tabular}{lccc}
\hline \multicolumn{1}{c}{ Variable } & \multicolumn{3}{c}{ Era } \\
\cline { 2 - 4 } & $\mathbf{1}$ & $\mathbf{2}$ & $\mathbf{3}$ \\
\hline Patients $(n)$ & 23 & 67 & 106 \\
\hline $\begin{array}{l}\text { Age at Dx (years) } \\
\text { Initial }\end{array}$ & & & \\
$\quad$ Median & & & 68 \\
$\quad$ Range & 66 & $23-87$ & $23-91$ \\
$\quad$ Metastatic \\
$\quad$ Median \\
$\quad$ Range
\end{tabular}


pre-imatinib era to 33.9 months (95\% CI: 16.6 months to 41.0 months; $p=0.044$ ) in the post-imatinib era, representing a median gain of 29.1 months free from progression in this population (Figure 3). In comparing the post-imatinib, pre-sunitinib era with the post-sunitinib era, a trend toward a slight decrease in PFS from 33.9 months to 28.0 months (95\% CI: 18.3 months to 41.9 months, $p=$ nonsignificant) was observed. Overall, median os increased by 54.4 months across all eras, and median PFS increased by 29.1 months between the first and second eras.

Although the ratio of men to women was non-equivalent in the various eras, no significant differences in survival outcomes based on sex were evident. A nonsignificant trend toward shorter os was observed for patients who presented with metastatic or unresectable disease at initial diagnosis compared with patients who went on to develop metastases after diagnosis (50.9 months vs. 63.5 months respectively; $95 \%$ CI: 41.1 months to 65.6 months; $p=0.2800$ ). Median PFS was equal regardless of whether the patient presented with unresectable or metastatic disease or developed metastatic disease later (28.3 months vs. 27.2 months respectively; $95 \%$ CI: 14.3 months to 35.5 months; $p=0.66)$. In our study population, median os $(p=0.2160)$ and median PFS ( $p=0.7459$ ) did not differ significantly for patients having high mitotic activity $\left(>5 / 5 \mathrm{~mm}^{2}\right)$ and low mitotic activity $\left(\leq 5 / 5 \mathrm{~mm}^{2}\right)$.

Univariate analysis revealed that primary site of disease, tumour size, and KIT status were significant predictors of PFS. Compared with patients having a primary tumour larger than $5 \mathrm{~cm}$ in the greatest dimension, those with a primary tumour less than $5 \mathrm{~cm}$ experienced significantly longer median PFS [55.8 months (95\% CI: 14.9 months to 130.9 months) vs. 26.6 months (95\% CI: 17.3 months to 33.8 months); $p=0.0293$ ]. Compared with their KIT-negative counterparts, patients with immunohistochemically confirmed KIT expression experienced significantly longer median PFS [33.8 months vs. 9.2 months (95\% CI: 0.9 months to 35.9 months); $p=0.0052$ ]. Compared with patients whose

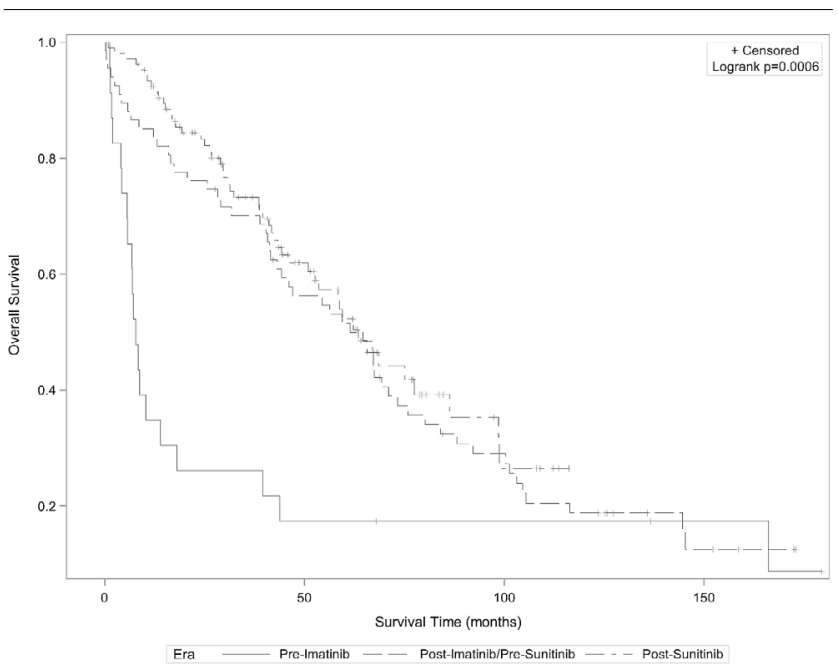

FIGURE 2 Kaplan-Meier survival curve comparing overall survival in the pre-imatinib (1996-2002), post-imatinib (2002-2007), and postsunitinib (2007-2016) eras. primary site of disease was the small intestine or all other locations, those with a primary site in the stomach experienced a significantly longer median PFs [35.6 months (95\% CI: 21.4 months to 55.8 months) vs. 22.6 months for small intestine (95\% CI: 10.4 months to 34.9 months) vs. 23.3 months for other (95\% CI: 7.0 months to 34.6 months), $p=0.0288$ ].

With respect to os, univariate analysis revealed that tumour size and KIT expression were significant predictors. In patients with a primary tumour larger than $5 \mathrm{~cm}$ in the greatest dimension, os was significantly shorter [50.9 months (95\% CI: 58.8 months to not estimable) vs. 98.6 months, $p=0.0081$ ). Compared with patients who expressed KIT protein, patients without KIT protein expression experienced significantly shorter os [ 16.5 months ( $95 \%$ CI: 10.7 months to 38.9 months) vs. 65.7 months, $p<0.0001]$. Of the 76 patients who underwent genetic analysis, $53(69.7 \%$ ) had a KIT mutation in exon 11; $15(19.7 \%)$ had a KIT mutation in exon 9, 13, or 17 ; and $8(10.5 \%)$ had no mutations that were tested for.

On multivariate analysis, primary tumour site and tumour size were confirmed to be predictive factors for survival. Compared with tumours smaller than $5 \mathrm{~cm}$ in the greatest dimension, those larger than $5 \mathrm{~cm}$ were associated with a much higher risk of an adverse event (hazard ratio for recurrence or death: 4.51; 95\% CI: 2.02 to 10.06). Risk of an adverse event was much lower for patients who received a TKI than for those who did not (hazard ratio: $4.70 ; 95 \%$ CI: 2.58 to 8.56$)$.

\section{DISCUSSION}

The implementation of TKI therapy in our non-trial population demonstrated a more dramatic improvement in OS and PFS than was estimated from the initial phase III studies upon which drug approval was based ${ }^{9-12}$. To our knowledge, the present study is the largest of its kind that has quantified the magnitude of the benefit from multiple TKIs on PFS and os in a non-trial setting over multiple decades. Median os was improved by 53.6 months, and

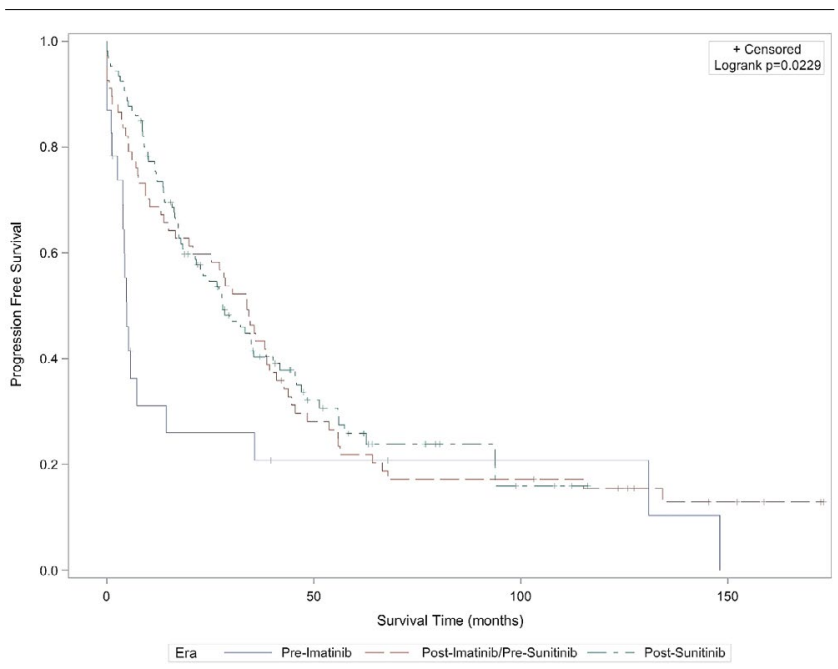

FIGURE 3 Kaplan-Meier survival curve comparing progression-free survival in the pre-imatinib (1996-2002), post-imatinib (2002-2007), and post-sunitinib (2007-2016) eras. 
median PFS was improved by 29.1 months for patients in the post-imatinib era. The subsequent availability of sunitinib did not result in any further significant improvements in the post-sunitinib era.

In our cohort study, TKI use was associated with a median os of 62.2 months and a median PFS of 33.9 months, which are substantially longer than the 48.8-month median OS and 20.0-month median PFS reported in the combined analysis of the phase III European Organisation for Research and Treatment of Cancer and swoG trials that led to the approval of imatinib ${ }^{9-12}$. Compared with patients in the pre-imatinib era, those in the post-sunitinib era experienced a significant increase in both os and PFs to 62.2 months and 28.0 months respectively. The results of the phase III clinical trial examining the efficacy of sunitinib in treating imatinib-resistant metastatic GIST showed a median OS and PFS of only 16.8 months and 5.3 months respectively ${ }^{23}$. It should be noted that survival outcomes in that particular phase III trial were calculated using a novel statistical analysis technique (rank-preserving structural failure time) to account for patient crossover from the placebo arm to the sunitinib arm. Using the Kaplan-Meier method, no statistically significant difference in os between the placebo and treatment arms was revealed, although os remained unchanged at 16.8 months $^{23}$. However, direct comparisons with our study are difficult to accurately estimate, because the reported values for the clinical trials involving imatinib and sunitinib were calculated using the date of randomization as the starting point.

Caution must be observed when applying clinical trial outcomes to the real-world setting, because clinical trials are carried out under standardized conditions and enrolment criteria often exclude patients with the worst prognoses. Trial criteria often lead to unrealistic expectations of treatment efficacy when applied to real-world use, frequently with an overestimation of the expected magnitude of the benefit of a new agent ${ }^{20}$. However, in the present study, we observed improvements in os and PFs for patients with metastatic GIST that were far better than were predicted by the results of the phase III randomized trials of imatinib. The incremental benefit with the addition of sunitinib was lower than expected, though.

It was interesting to note the slight reduction in PFS of 5.9 months between the post-imatinib and post-sunitinib eras. Although statistically insignificant, that finding is relatively misleading. In our cohort, most patients received sunitinib only after progression on first-line imatinib (96\% of the patients who received sunitinib). As a result, PFs in the final era cannot be used as a direct indicator of the cumulative benefit of sunitinib; it is instead indicative of the efficacy of imatinib treatment in that era. A greater willingness to administer imatinib to patients with a worse prognosis or greater comorbidities might have been responsible for the slight decrease in median PFS observed in the post-sunitinib cohort. Regardless, the introduction of imatinib and sunitinib for the treatment of metastatic GIST has proved to be a major breakthrough for improving patient outcomes.

In 2006, based on a study of 1765 patients, Miettinen et al. ${ }^{24}$ developed a risk classification system for localized GISTs that listed tumour size, mitotic activity, and primary tumour site as the 3 main predictors of recurrence or metastases. Upon univariate analysis in our study of patients with metastatic disease, only tumour size, primary tumour site, and KIT expression were significant predictors of survival outcomes. Multivariate analysis showed that primary site predicted survival to a lesser magnitude than did tumour size and the advent of TKIs. Patients with gastric GISTs were found to have the highest PFS; those with small intestine GISTs had the lowest PFS (stomach: 35.6 months; small intestine: 22.6 months; other: 23.2 months). That observation was consistent with the results of several studies that have found that GISTs originating in the stomach carry the best prognosis and that those arising in the small intestine carry the worst prognosis ${ }^{25-27}$. A lack of KIT expression and a primary tumour size larger than $5 \mathrm{~cm}$ were both associated with a substantial reduction in both os and PFS. Because imatinib is indicated for use only in KIT-positive GISTs, the shorter survival outcomes for patients who are KIT-negative might be attributable to a lack of available and effective targeted therapies. Still, several studies have suggested that a subset of patients with CD117-negative disease might harbour imatinib-sensitive mutations in KIT or PDGFRA ${ }^{28-31}$. The lack of an evidence-based consensus about the efficacy of imatinib in treating CD117-negative incurable GISTs warrants future research.

The limitations of accuracy imposed by the method of standardization for mitotic activity are recognized as an unavoidable consequence of this retrospective review. A 2010 review of risk reporting in GISTs found that counting mitoses in 10 HPFs before calculating the ratio in 50 HPFs could lead to false results, because mitotic activity is often counted in the area in which mitotic figures are most numerous $^{32}$. Given the retrospective nature of our study, tumour characteristics such as KIT and PDGFRA mutational status were left unreported for a substantial proportion of the study population. Although KIT expression was not tested in most patients in the pre-imatinib era, it is unlikely that results would have differed greatly from those in the rest of the study population, and most patients would presumptively have been KIT-positive according to findings in previous studies? Despite the lack of complete patient information, it is unlikely that the dramatic survival outcome improvements in the patient cohorts were the result of non-recorded factors.

Minor advances in imaging and supportive care might have elicited a small positive effect on patient survival during the study eras. Still, no significant advances in the clinical diagnosis of GIST were put into practice between the time of the phase III clinical trials discussed earlier and the time of this paper being written. In addition, referral bias might have been present before the advent of TKIs. Patients with metastatic GIST might not have been referred to an oncologist because of poor prognosis and a lack of available treatment options, thus underestimating the true effect on patient survival of introducing TKIs. Overall, we do not believe that those confounding variables have substantially affected our findings.

\section{CONCLUSIONS}

The use of imatinib in the setting of metastatic GIST has prolonged life by 53.6 months for affected patients in British Columbia - a result that is preserved across all prognostic 
subgroups. The further addition of sunitinib marginally improved os. Further work to evaluate resistance pathways to TKIs in this population might help to inform further therapeutic strategies.

To our knowledge, this study is the largest of its kind to evaluate the real-world incremental benefit of imatinib and sunitinib by comparing outcomes of patients in the pre-imatinib era with those of patients in the postimatinib, pre-sunitinib era and the post-sunitinib era. As more targeted therapies are released for use in metastatic GIST, it will be important to evaluate the effect on patient outcomes of administering multiple lines of TKIs, considering the high likelihood of resistance mechanisms arising in tumour cells.

The findings of our study play an important role in the ability to convey to patients the improvement and impact of TKIs on the natural history of metastatic GIST. In the treatment of this disease, implementation of TKIs in clinical practice has outperformed use of those agents in clinical trials.

\section{ACKNOWLEDGMENTS}

We acknowledge Maya Chu and her family for generous donations that have supported the Sarcoma Outcomes Unit at BC Cancer. An abstract of this manuscript was previously presented in poster form at the Connective Tissue Oncology Society (СтоS) 2017 annual meeting and the Canadian Cancer Research Conference 2017 conference. The abstract (poster 066) is available online in the CTOS meeting program at https://www.ctos.org/Portals/0/ PDF/2017\%20CTOS\%20Final\%20Program.pdf.

\section{CONFLICT OF INTEREST DISCLOSURES}

We have read and understood Current Oncology's policy on disclosing conflicts of interest, and we declare that we have none.

\section{AUTHOR AFFILIATIONS}

${ }^{*}$ Medical Oncology, ${ }^{\dagger}$ Surgical Oncology, and ${ }^{\ddagger}$ Statistical Sciences, BC Cancer, Vancouver, BC; ${ }^{\S}$ Medical Oncology, oHsu Knight Cancer Institute, Portland, OR, U.S.A.

\section{REFERENCES}

1. Zhao X, Yue C. Gastrointestinal stromal tumor.J Gastrointest Oncol 2012;3:189-208.

2. Søreide K, Sandvik OM, Søreide JA, Giljaca V, Jureckova A, Bulusu VR. Global epidemiology of gastrointestinal stromal tumors (GIST): a systematic review of population-based cohort studies. Cancer Epidemiol 2016;40:39-46.

3. Dematteo RP, Heinrich MC, El-Rifai WM, Demetri G. Clinical management of gastrointestinal stromal tumors: before and after STI-571. Hum Pathol 2002;33:466-77.

4. Knowlton CA, Brady LW, Heintzelman RC. Radiotherapy in the treatment of gastrointestinal stromal tumor. Rare Tumors 2011;3:e35.

5. Rock EP, Goodman V, Jiang JX, et al. Food and Drug Administration drug approval summary: sunitinib malate for the treatment of gastrointestinal stromal tumor and advanced renal cell carcinoma. Oncologist 2007;12:107-13.

6. Hirota S, Isozaki K, Moriyama Y, et al. Gain-of-function mutations of $c$-KIT in human gastrointestinal stromal tumors. Science 1998;379:577-80.

7. Corless CL, Heinrich MC. Molecular pathobiology of gastrointestinal stromal sarcomas. Annu Rev Pathol 2008;3:557-86.

8. Heinrich MC, Corless CL, Duensing A, et al. PDGFRA activating mutations in gastrointestinal stromal tumors. Science 2003;299:708-10.
9. Cohen MH, Farrell A, Justice R, Pazdur R. Approval summary: imatinib mesylate in the treatment of metastatic and/ or unresectable malignant gastrointestinal stromal tumors. Oncologist 2009;14:174-80.

10. Blanke CD, Rankin C, Demetri GD, et al. Phase III randomized, Intergroup trial assessing imatinib mesylate at two dose levels in patients with unresectable or metastatic gastrointestinal stromal tumors expressing the KIT receptor tyrosine kinase: S0033. J Clin Oncol 2008;26:626-32.

11. Verweij J, Casali PG, Zalcberg J, et al. Progression-free survival in gastrointestinal stromal tumours with high-dose imatinib: randomized trial. Lancet 2004;364:1127-34.

12. Gastrointestinal Stromal Tumor Meta-Analysis Group. Comparison of two doses of imatinib for the treatment of unresectable or metastatic gastrointestinal stromal tumors: a meta-analysis of 1,640 patients. JClin Oncol 2010;28:1247-53.

13. BC Cancer. BC Cancer Protocol Summary for Treatment of Advanced c-KIT Positive and c-KIT Negative Gastrointestinal Stromal Cell Tumours (GISTs) Using Imatinib. Vancouver, BC: BC Cancer; 2018. [Available online at: http://www.bccancer. bc.ca/chemotherapy-protocols-site/Documents/Sarcoma/ SAAVGI_Protocol.pdf; cited 10 August 2019]

14. Kee D, Zalcberg JR. Current and emerging strategies for the management of imatinib-refractory advanced gastrointestinal stromal tumors. Ther Adv Med Oncol 2012;4:255-70.

15. Wang CM, Huang K, Zhou Y, et al. Molecular mechanisms of secondary imatinib resistance in patients with gastrointestinal stromal tumors. J Cancer Res Clin Oncol 2010;136:1065-71.

16. Demetri GD, van Oosterom AT, Garrett CR, et al. Efficacy and safety of sunitinib in patients with advanced gastrointestinal stromal tumor after failure of imatinib: a randomized controlled trial. Lancet 2006;368:1329-38.

17. BC Cancer. BCCancer Protocol Summary for Second Line Treatment of Advanced c-KIT Positive Gastrointestinal Stromal Cell Tumours (GIST) after Imatinib Using Sunitinib. Vancouver, BC: BC Cancer; 2019. [Available online at: http://www.bccancer. bc.ca/chemotherapy-protocols-site/Documents/Sarcoma/ SAAVGS_Protocol.pdf; cited 10 August 2019]

18. Ferraro D, Zalcberg J. Regorafenib in gastrointestinal stromal tumors: clinical evidence and place in therapy. Ther Adv Med Oncol 2014;6:222-8.

19. Barrios CH, Blackstein ME, Blay JY, et al. The Gold Registry: a global, prospective, observational registry collecting longitudinal data on patients with advanced and localised gastrointestinal stromal tumours. Eur J Cancer 2015;51:2423-33.

20. Martin K, Bégaud B, Latry P, Miremont-Salame G, Fourrier A, Moore N. Differences between clinical trials and marketing use. Br J Clin Pharmacol 2004;57:86-92.

21. Sarkar RR, Matsuno R, Murphy JD. Pancreatic cancer: survival in clinical trials versus the real world [abstract 216]. J Clin Oncol 2016;34:. [Available online at: https://ascopubs.org/ doi/abs/10.1200/jco.2016.34.4_suppl.216; cited 26 May 2020]

22. Flather M, Delahunty N, Collinson J. Generalizing results of randomized trials to clinical practice: reliability and cautions. Clin Trials 2006;3:508-12.

23. Demetri GD, Garrett CR, Schöffski P, et al. Complete longitudinal analyses of the randomized, placebo-controlled, phase III trial of sunitinib in patients with gastrointestinal stromal tumor following imatinib failure. Clin Cancer Res 2012;18:3170-9.

24. Miettinen M, Sobin LH, Lasota J. Gastrointestinal stromal tumors of the stomach: a clinicopathologic immunohistochemical, and molecular genetic study of 1765 cases with long-term follow-up. Am J Surg Pathol 2005;29:52-68.

25. Miettinen M, Lasota J. Gastrointestinal stromal tumors: pathology and prognosis at different sites. Semin Diagn Pathol 2006;23:70-83. 
26. Emory TS, Sobin LH, Lukes L, Lee DH, O'Leary TJ. Prognosis of gastrointestinal smooth-muscle (stromal) tumors: dependence on anatomic site. Am J Surg Pathol 1999;23:82-7.

27. Stamatakos M, Douzinas E, Stefanaki C, et al. Gastrointestinal stromal tumor. World J Surg Oncol 2009;7:61.

28. Medeiros F, Corless CL, Duensing A, et al. KIT-negative gastrointestinal stromal tumors: proof of concept and therapeutic implications. Am J Surg Pathol 2004;28:889-94.

29. Blackstein ME, Blay JY, Corless C, et al. Gastrointestinal stromal tumours: consensus statement on diagnosis and treatment. Can J Gastroenterol 2006;20:157-64.
30. Heinrich MC, Griffith DJ, Druker BJ, Wait CL, Ott KA, Zigler AJ. Inhibition of c-KIT receptor tyrosine kinase activity by STI 571, a selective tyrosine kinase inhibitor. Blood 2000;96:925-32.

31. Corless CL, Schroeder A, Griffith D, et al. PDGFRA mutations in gastrointestinal stromal tumors: frequency, spectrum and in vitro sensitivity to imatinib. J Clin Oncol 2005;23:5357-64.

32. Agaimy A. Gastrointestinal stromal tumors (GIST) from risk stratification systems to the new TNM proposal: more questions than answers? A review emphasizing the need for a standardized GIST reporting. Int J Clin Exp Pathol 2010; $3: 461-71$ 